\title{
Detection and enumeration of periodontopathogenic bacteria in subgingival biofilm of pregnant women
}

\section{Fernanda Campos Machado(a) Dionéia Evangelista Cesar ${ }^{(b)}$ Amanda Vervloet Dutra Agostinho Assis $^{(c)}$ \\ Cláudio Galuppo Diniz (d) Rosangela Almeida Ribeiro(c)}

\footnotetext{
(a) Postgraduate Program in Health, School of Medicine, Univ Federal de Juiz de Fora UFJF, Juiz de Fora, MG, Brazil.

(b) Department of Ecology and Molecular Biology of Microorganisms, Biological Sciences Institute, Univ Federal de Juiz de Fora - UFJF, Juiz de Fora, MG, Brazil.

(c) Department of Social and Child Dentistry, School of Dentistry, Univ Federal de Juiz de Fora - UFJF, Juiz de Fora, MG, Brazil.

(d) Department of Parasitology, Microbiology and Immunology, Biological Sciences Institute, Univ Federal de Juiz de Fora UFJF, Juiz de Fora, MG, Brazil.
}

\begin{abstract}
The aim of this study was to use the fluorescence in situ hybridization (FISH) technique to test the hypothesis of qualitative and quantitative differences of 8 periodontopathogens between pregnant and non-pregnant women. This cross-sectional study included 20 pregnant women in their second trimester of pregnancy and 20 non-pregnant women. Probing depth, bleeding on probing, clinical attachment level, and presence of calculus were recorded. Subgingival plaque samples were collected and the FISH technique identified the presence and numbers of Aggregatibacter actinomycetemcomitans, Tannerella forsythia, Campylobacter rectus, Porphyromonas gingivalis, Treponema denticola, Fusobacterium nucleatum, Prevotella intermedia and Prevotella nigrescens. The Mann-Whitney $U$-test was applied to compare the data between the two groups. The mean age, ethnicity, marital status, education, and economic level in both groups were similar. The clinical parameters showed no significant differences between pregnant and non-pregnant women. The numbers of subgingival periodontopathogens were not found to be significantly different between groups, despite the higher mean counts of $P$. intermedia in pregnant women. Colonization patterns of the different bacteria most commonly associated with periodontal disease were not different in the subgingival plaque of pregnant and non-pregnant women.
\end{abstract}

Descriptors: Pregnancy; Microbiology; Biofilms; In situ Hybridization, Fluorescence.

\section{Introduction}

The hormonal changes in pregnant women appear to be correlated with the enhanced growth of certain Gram-negative anaerobic bacteria in the oral cavity, stimulating the growth of Prevotella intermedia in particular. ${ }^{1-3}$ Higher levels of Campylobacter rectus and Prevotella nigrescens have also been found among these women. ${ }^{4,5}$ However, this issue remains controversial.

In addition, during pregnancy, other periodontal pathogens, such as Tannerella forsythia, Porphyromonas gingivalis, Aggregatibacter actinomycetemcomitans, and Treponema denticola, should also be taken into account because of the relationship between certain subgingival species and preterm birth..$^{6-8}$ Further studies are needed to identify the etiologic factors that might explain this relationship. ${ }^{9}$

In previous studies with pregnant women, periodontopathogens
Submitted: Mar 15, 2012

Accepted for publication: Jun 05, 2012

Last revision: Jun 17, 2012 
have been identified by traditional anaerobic culture methods, PCR-based methods, or DNA-DNA hybridization. ${ }^{1-5,10,11}$ About $50 \%$ of oral bacterial strains cannot be cultured by conventional methods. For some periodontopathogens, molecular techniques, such as PCR, real-time PCR, or DNA-DNA hybridization, have been used successfully to detect microorganisms that are difficult to culture. ${ }^{4,5,9}$ Nonetheless, these methods are only qualitative or semi-quantitative, or are used to quantify the numbers of amplified copies of genes and/or gene expression, and thereby do not provide information about the morphology, specific numbers, and spatial distribution of microorganisms. In this context, one technique that could be used is fluorescence in situ hybridization (FISH) with synthetic ribosomal RNA (rRNA)-target oligonucleotide probes. In this technique, the cell remains intact and each individual cell can be counted. ${ }^{12,13}$

The FISH technique could provide accurate information on periodontal microbiota in normal pregnancy, to improve our knowledge of the relationship between pregnancy and periodontal disease and to help identify the microbiota that might be linked to risks for birth complications.

We conducted the present study using the FISH technique to test the hypothesis of qualitative and quantitative differences of $A$. actinomycetemcomitans, T. forsythia, C. rectus, P. gingivalis, T. denticola, Fusobacterium nucleatum, P. intermedia and $P$. nigrescens between pregnant and non-pregnant women.

\section{Methodology}

We adopted a cross-sectional research design and identified a convenience sample. The study was approved by the Ethics Committee in Human Research of the Federal University of Juiz de Fora (UFJF), MG, Brazil (protocol number 130/2010). All participants signed an informed consent form. The present data represent cases enrolled between June and December 2010. Expectant mothers were recruited during routine prenatal health visits, and the non-pregnant women were recruited from the Reception Department of the School of Dentistry of UFJF.
Information about age, marital status, education and economic levels, and medical history was assessed by interview.

\section{Study population}

The pregnant group in the study population ( $\mathrm{Pr}$ group) was comprised of 20 consecutive women from 24 to 32 years old at gestational stage between 14 and 24 weeks. Gestational age was confirmed by ultrasound. Twenty non-pregnant women within the same age range were recruited as controls (N-Pr group).

All participants were in good general health and had a minimum of 20 permanent teeth. Women with chronic disease, smokers, alcohol dependency, reported use of systemic antibiotics and/or psychotropic or anticonvulsant medication in the preceding 3 months, as well as those who required prophylactic antibiotics for periodontal examination or who had received professional tooth-cleaning in the preceding 6 months, or who were orthodontic patients, were excluded.

\section{Periodontal examination}

Full-mouth data were recorded according to the following clinical parameters:

- probing depth (PD),

- bleeding on probing (BOP), ${ }^{14}$

- clinical attachment level (CAL) and

- presence of calculus (PC).

All teeth were examined, with the exception of third molars, by means of a North Carolina periodontal probe (PCP-UNC 15, Hu-Friedy Manufacturing Inc., Chicago, USA). Six sites per tooth were examined, and the highest scores of PD, CAL, BOP, and $\mathrm{PC}$ were registered for each tooth. Periodontal examination was conducted by a single trained and calibrated dentist (FCM). Intra-examiner agreement showed Cohen's Kappa coefficients of 0.86 for PD and 0.90 for CAL.

Periodontal disease was defined as the presence of 4 or more teeth with 1 or more sites of PD $\geq 4 \mathrm{~mm}$ and $\mathrm{CAL} \geq 3 \mathrm{~mm}$ at the same site. ${ }^{15}$ 


\section{Microbiological analysis}

The plaque sampling followed the protocol established by Madianos et al. ${ }^{8}$ Supragingival biofilm was removed with sterile gauze, and subgingival biofilm samples were collected from the mesial surfaces of 2 opposite and contralateral first molars (or, if missing, the next most posterior tooth) by means of sterile Gracey curettes (Hu-Friedy Manufacturing Inc.). The biofilm samples were transferred into a $1.5-\mathrm{mL}$ screw-cap microtube and immediately transported on dry ice to the laboratory.

The microtubes were weighed before and immediately after the sampling, to standardize the collection and facilitate the quantitative analysis. Samples were fixed and stored in paraformaldehyde solution, $2 \%$ final concentration. The material was kept in a freezer at $-20^{\circ} \mathrm{C}$ until it was used for microbiological analysis.

The FISH technique was carried out to identify and enumerate the periodontal pathogens in the subgingival biofilm of the women. Eight speciesspecific 16S rRNA oligonucleotide probes (Operon Technologies Inc., Alameda, USA), labeled with Cy3 fluorescent dye, were used (Table 1). ${ }^{16-20}$

A negative control probe (5'-CCTAGTGACGCCGTCGAC-3'), with a sequence that should not bind to any prokaryote rRNA, was used. ${ }^{21}$

The samples were prepared by sonication and centrifugation, ${ }^{22}$ after which a $5-\mathrm{mL}$ sample was filtrated into $0.2-\mu \mathrm{m}$ membrane filters.

FISH was performed according to a method described by Cottrel and Kirchman. ${ }^{21}$ The filters were divided into 9 equal pieces. Each piece was placed on a slide, overlaid with a hybridization solution at a final concentration of $2.5 \mathrm{ng} / \mu \mathrm{L}$ of Cy3-labeled oligonucleotide probe, and incubated in a sealed container overnight at $42^{\circ} \mathrm{C}$. The hybridization solution contained $0.9 \mathrm{M} \mathrm{NaCl}, 20 \mathrm{mM}$ Tris- $\mathrm{HCl}(\mathrm{pH}$ 7.4), $0.01 \%$ sodium dodecyl sulfate, and the concentration of formamide determined to achieve specificity for each targeted group of bacteria (Table 1). After hybridization, the sample was transferred to a washing solution and stained with $2 \mu \mathrm{g}$ of DAPI (4',6'-diamidino-2-phenylindole) per $\mathrm{mL}$, so that the bacterial cells could be counted.

Total bacterial cells and cells from each species were counted by means of an Olympus BX60 microscope fitted with a DAPI filter 31000 and Cy3 filter 41007a (Chroma, Bellows Falls, USA), respectively. The numbers of cells observed in 10 randomized microscopic ocular grid fields per sample were counted by a single trained observer (FCM). The final number of bacteria was calculated from the multiplication of the dilutions performed throughout the treatment of the sample, based on the initial weight. Results are presented in cells/g.

For documentation, photomicrographs were taken with an Evolution VF Color Cooled camera (Media Cybernetics Inc., Bethesda, USA) attached to the microscope.

\section{Statistical analysis}

The statistical analysis was performed with SPSS 14.0 (SPSS Inc., Chicago, USA). Nominal data were described by relative and/or absolute frequencies. Numerical data were described by mean, standard

Table 1 - Sequences of 16S rRNA oligonucleotide probes and formamide concentrations for fluorescence in situ hybridization.

\begin{tabular}{|c|c|c|c|c|}
\hline Probe & Target species & Sequence $\left(5^{\prime}-3^{\prime}\right)$ & Formamide (\%) & Reference \\
\hline ACAC & A. actinomycetemcomitans & TCCATAAGACAGATTC' & 30 & Sunde et al. ${ }^{16}$ \\
\hline $\mathrm{B} / \mathrm{TAFO}$ & T. forsythia & CGTATCTCATTTTATTCCCCTGTA & 30 & Sunde et al. ${ }^{16}$ \\
\hline CARE & C. rectus & TTAACTTATGTAAAGAAG & 20 & Riep et al. ${ }^{17}$ \\
\hline POGI & P. gingivalis & CAATACTCGTATCGCCCGTTATTC & 30 & Sunde et al. ${ }^{16}$ \\
\hline TRE II & T. denticola & GCTCCTTTCCTCATTTACCTTTAT' & 30 & Moter et al. ${ }^{18}$ \\
\hline FUS664 & F. nucleatum & CTTGTAGTTCCG C(C/T)TACCTC & 40 & Gmür et al. ${ }^{19}$ \\
\hline Pint649 & P. intermedia & GCCGCCRCTGAASTCAAGCC & 40 & Gmür and Thurnheer ${ }^{20}$ \\
\hline Pnig657 & P. nigrescens & TCCGCCTGCGCTGCGTGTA & 40 & Gmür and Thurnheer ${ }^{20}$ \\
\hline
\end{tabular}


deviation, and minimum and maximum values. The non-parametric Mann-Whitney $U$-test was used for data comparison between the Pr and N-Pr groups. Data were considered as statistically significant at $p$ values $<0.05$.

\section{Results}

The mean age of the pregnant women was 26.4 years (SD \pm 2.5 ), while that of the non-pregnant women was 27.4 years ( $\mathrm{SD} \pm 2.2$ ). In the Pr group, the mean gestational age was 18.9 weeks ( $\mathrm{SD} \pm 3.4$ ).

No significant differences were observed between the Pr and N-Pr groups in terms of age $(p=0.584)$, ethnicity $(p=0.393)$, marital status $(p=0.251)$, education $(p=0.478)$, and economic level $(p=0.315)$.

\section{Periodontal status}

Both groups had similar periodontal conditions. There were no significant differences between $\mathrm{Pr}$ and N-Pr groups in numbers of teeth and their clinical parameters (Table 2). Among the Pr and N-Pr groups, 14 and 16 women, respectively, had no periodontal disease $(p=0.256)$.

\section{Microbiological results}

The mean total bacterial count was $492 \times 10^{7} \pm 233 \times 10^{7}$ cells/g and $624 \times 10^{7} \pm$ $301 \times 10^{7}$ cells/g for the Pr and N-Pr groups, respectively. Statistical analysis demonstrated no significant difference between groups $(p=0.13)$.

Analysis by the Mann-Whitney $U$-test failed to demonstrate significant differences between groups in the numbers of all bacterial species evaluated $(\mathrm{Ta}-$ ble 3).

Figure 1 shows a field from a representative plaque sample stained for total bacterial cells (with DAPI) and for P. intermedia (with probe Pint649). The images demonstrate the presence of these bacte-

Table 2 - Clinical periodontal parameters of pregnant and nonpregnant women

\begin{tabular}{c|c|c|c}
\hline \multirow{2}{*}{ Variable } & Pr group $(\mathrm{n}=20)$ & N-Prgroup $(\mathrm{n}=20)$ & \multirow{2}{*}{ p value } \\
\cline { 2 - 3 } & Mean \pm SD & Mean \pm SD & \\
\hline Mean number of teeth & $26.7 \pm 1.3$ & $26.1 \pm 2.2$ & 0.53 \\
\hline Teeth with BOP (\%) & $48.1 \pm 30.9$ & $49.3 \pm 29.2$ & 0.79 \\
\hline Teeth with PC (\%) & $12.3 \pm 11.9$ & $13.4 \pm 20.2$ & 0.61 \\
\hline Teeth with PD $=4-5 \mathrm{~mm}(\%)$ & $6.3 \pm 15.4$ & $1.7 \pm 4.1$ & 0.55 \\
\hline Teeth with PD $\geq 6 \mathrm{~mm}(\%)$ & $0.9 \pm 3.3$ & $0.2 \pm 0.9$ & 0.53 \\
\hline Teeth with CAL $=3-5 \mathrm{~mm}(\%)$ & $1.2 \pm 3.3$ & 0 & 0.15 \\
\hline
\end{tabular}

${ }^{*} \mathrm{BOP}=$ bleeding on probing; $\mathrm{PC}=$ presence of calculus; $\mathrm{PD}=$ probing depth; $\mathrm{CAL}=$ clinical attachment level; $\mathrm{SD}=$ standard deviation; $\mathrm{n}=$ number.

Table 3 - Mean, standard deviation (SD), and minimum and maximum values of the counts of periodontal pathogens from pregnant (Pr group) and non-pregnant (N-Pr group) women.

\begin{tabular}{c|c|c|c|c|c|c|c}
\hline \multirow{2}{*}{$\begin{array}{c}\text { Periodontal pathogen } \\
\left(\text { cells } \times 10^{7} / \mathrm{g}\right)\end{array}$} & \multicolumn{3}{|c|}{$\operatorname{Pr}$ group $(\mathrm{n}=20)$} & \multicolumn{3}{c}{ N-Prgroup $(\mathrm{n}=20)$} \\
\cline { 2 - 8 } & Mean \pm SD & Minimum & Maximum & Mean \pm SD & Minimum & Maximum \\
\hline A. actinomycetemcomitans & $12 \pm 13$ & 0.8 & 56 & $19 \pm 25$ & 0 & 89 & 0.95 \\
\hline T. forsythia & $26 \pm 22$ & 2.5 & 75 & $28 \pm 23$ & 7.0 & 85 & 0.63 \\
\hline C. rectus & $16 \pm 16$ & 0 & 54 & $31 \pm 47$ & 0 & 143 & 0.79 \\
\hline P. gingivalis & $25 \pm 41$ & 0 & 181 & $22 \pm 21$ & 0 & 81 & 0.75 \\
\hline T. denticola & $18 \pm 16$ & 0 & 59 & $19 \pm 22$ & 0 & 88 & 0.79 \\
\hline F. nucleatum & $26 \pm 30$ & 0 & 133 & $40 \pm 30$ & 0 & 102 & 0.07 \\
\hline P. intermedia & $54 \pm 107$ & 0 & 474 & $35 \pm 30$ & 2.0 & 98 & 0.34 \\
\hline P. nigrescens & $30 \pm 28$ & 0 & 116 & $34 \pm 32$ & 0 & 131 & 0.74 \\
\hline
\end{tabular}



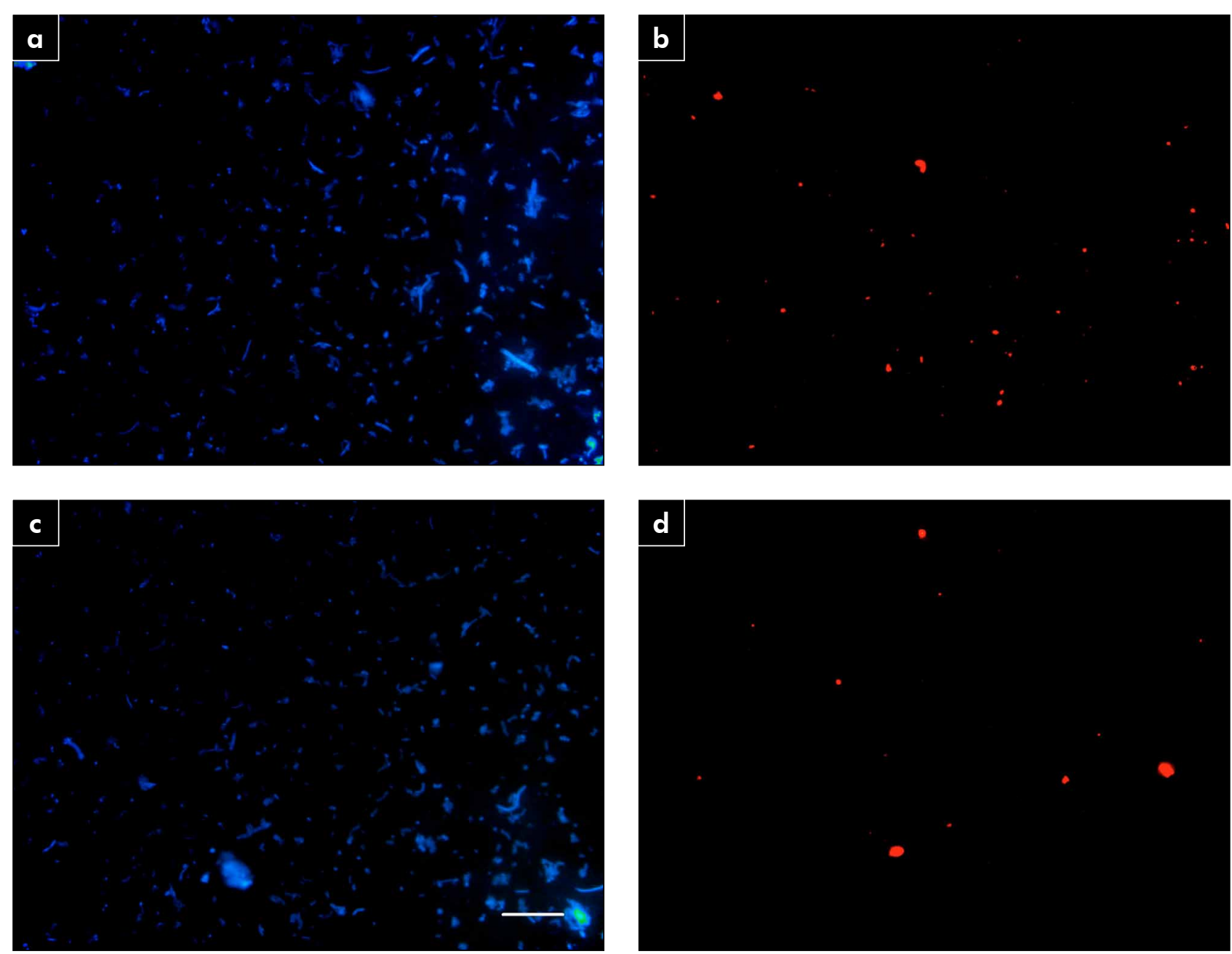

Figure 1 - DAPI staining for total bacterial cell counts and $P$. intermedia of subgingival plaque samples from pregnant (a, b) and non-pregnant (c, d) women. The scale bar indicates $20 \mu \mathrm{m}$.

rial cells in pregnant and non-pregnant women.

\section{Discussion}

To our knowledge, this is the first study that has used the FISH technique to assess periodontopathogens during pregnancy.

All women included in the present study were from 24 to 32 years old, and most had no periodontal disease. Both groups were similar in terms of clinical periodontal parameters and were matched by age, ethnicity, marital status, education, and economic level. In older people, periodontal disease is likely to be more pronounced. ${ }^{23}$ Also, different specific stages in life, such as puberty and menopause, can modulate periodontal tissue response and therefore contribute to periodontal disease. ${ }^{24}$ Addition- ally, oral health status could also be related to the level of education, ethnicity, marital status, and economic level. ${ }^{23}$ The young mean age of this study population, together with the similarities in the socio-demographic variables and clinical periodontal parameters, might have resulted in the absence of differences in terms of the composition of the subgingival microbiota.

Although exacerbated gingival inflammation in pregnant women has been clinically and histologically well-documented, its aetiology has not yet been clearly established, and it is not known why only some pregnant women develop frank signs of gingival inflammation. ${ }^{11,25}$ Changes in the sub- or supragingival biofilm ${ }^{1-3}$ have been proposed as a potential mechanism; however, there are only limited 
data on the composition of the subgingival microbiota during pregnancy.

An increase in $P$. intermedia in the subgingival biofilm of pregnant women during the second trimester was first reported in $1980 \cdot^{1-3}$ In our study, elevated counts of $P$. intermedia were observed in the Pr-group; however, no significant differences were seen. Other investigators also showed no significant microbiological differences between pregnant and non-pregnant women. ${ }^{5,10}$

Differences in methods used to assess bacteria may partly explain the results reported. In most cases, the previously mentioned studies ${ }^{1-3,10,11}$ used classic microbiological analyses, such as culture methods, which are hampered by the complexity of the periodontal microbiota and the fastidious nature of these microorganisms. ${ }^{11,13}$ These methods can also be laborious, time-consuming and prone to statistical and methodological errors. ${ }^{13}$ More recently, techniques that do not require previous culturing, like PCR-based assays and DNA-DNA hybridization, have been used to detect periodontal pathogens in pregnant women, ${ }^{4,5,9}$ but so far they have the disadvantage of yielding only qualitative or semiquantitative data. ${ }^{13,20}$ FISH combines the precision of molecular genetics with the visual information from microscopy, allowing direct visualization and identification of individual cells within their natural microhabitat or diseased tissue. ${ }^{13}$ Additionally, this technique showed a high standard deviation and a high variability of bacterial loads in this population by the count of absolute values, not only by the presence or absence of microorganisms, as with other molecular techniques.

Increasing evidence is accumulating that oral bacteria may translocate directly into the pregnant uterus, causing localized inflammation and adverse pregnancy outcomes, notwithstanding the presence of clinical periodontitis. ${ }^{26} \mathrm{~F}$. nucleatum, $P$. gingivalis, A. actinomycetencomitans, T. denticola, C. rectus, and T. forsythia are examples of such species. ${ }^{6-8}$ The high levels of these pathogens observed in pregnant women in the present study, independent of the presence of periodontitis or the lack of differences in comparison with non-pregnant women, may be suggested as an increased risk of adverse pregnancy outcomes. Early diagnosis and extra care in control of these microorganisms might be necessary.

One can imagine that levels of periodontal bacteria would vary according to gestational phase. Nevertheless, previous studies have shown that the highest proportions of periodontal pathogens and the major changes in clinical parameters occur in the second trimester of pregnancy, ${ }^{2,5,9}$ which was an inclusion criterion for this study. Continued longitudinal studies with the FISH technique would enable investigators to verify the actual subgingival microbiological patterns during pregnancy and post partum. In addition, the periodontal pathogens in other samples, e.g., saliva or gingival crevicular fluid, should be investigated by FISH.

Furthermore, we do not ignore the fact that periodontal microbiological patterns could be associated with plaque index, which was not recorded in our study. Despite that limitation, the results of this study of pregnant and non-pregnant women provide additional insights into their periodontal microbiota, identified and quantified by the FISH technique.

The fact that the subgingival microbiota in healthy pregnant women did not differ from that in the non-pregnant women may suggest that the hormonal changes in pregnancy exert little, if any, influence on colonization/growth patterns of the 8 different bacteria most commonly associated with periodontal disease, at least in women with little evidence of periodontitis.

\section{Conclusions}

The study did not confirm the hypothesis of qualitative and quantitative differences, between pregnant and non-pregnant women, in terms of the 8 periodontopathogens evaluated by the FISH technique.

\section{Acknowledgements}

The authors thank Dr. Luiz Cláudio Ribeiro (Department of Statistics, UFJF, Brazil) for assistance with the statistical analysis. This study was supported by research grants from CAPES, Brasilia, Brazil, as well as by grants from the Postgraduate Program in Health of UFJF, Brazil. 


\section{References}

1. Jensen J, Liljemark W, Bloomquist C. The effect of female sex hormones on subgingival plaque. J Periodontol. 1981 Oct;52(10):599-602.

2. Kornman KS, Loesche WJ. The subgingival microbial flora during pregnancy. J Periodontal Res. 1980 Mar;15(2):111-22.

3. Muramatsu Y, Takaesu Y. Oral health status related to subgingival bacterial flora and sex hormones in saliva during pregnancy. Bull Tokyo Dent Coll. 1994 Aug;35(3):139-51.

4. Yokoyama M, Hinode D, Yoshioka M, Fukui M, Tanabe S, Grenier D, et al. Relationship between Campylobacter rectus and periodontal status during pregnancy. Oral Microbiol Immunol. 2008 Feb;23(1):55-9.

5. Gürsoy M, Haraldsson G, Hyvönen M, Sorsa T, Pajukanta R, Könönen E. Does the frequency of Prevotella intermedia increase during pregnancy?. Oral Microbiol Immunol. 2009 Aug;24(4):299-303.

6. Hasegawa K, Furuichi Y, Shimotsu A, Nakamura M, Yoshinaga M, Kamitomo M, et al. Associations between systemic status, periodontal status, serum cytokine levels, and delivery outcomes in pregnant women with a diagnosis of threatened premature labor. J Periodontol. 2003 Dec;74(12):1764-70.

7. Offenbacher S, Jared HL, O'Reilly PG, Wells SR, Salvi GE, Lawrence HP, et al. Potential pathogenic mechanisms of periodontitis associated pregnancy complications. Ann Periodontol. 1998 Jul;3(1):233-50.

8. Madianos PN, Lieff S, Murtha AP, Boggess KA, Auten RL, Beck JD, et al. Maternal periodontitis and prematurity. Part II: Maternal infection and fetal exposure. Ann Periodontol. 2001 Dec;6(1):175-82.

9. Adriaens LM, Alessandri R, Sporri S, Lang NP, Persson GR. Does pregnancy have an impact on the subgingival microbiota?. J Periodontol. 2009 Jan;80(1):72-81.

10. Jonsson R, Howland BE, Bowden GH. Relationships between periodontal health, salivary steroids, and Bacteroides intermedius in males, pregnant and non-pregnant women. J Dent Res. 1988 Aug;67(8):1062-9.

11. Carrillo-de-Albornoz A, Figuero E, Herrera D, BasconesMartinez A. Gingival changes during pregnancy: II. Influence of hormonal variations on the subgingival biofilm. J Clin Periodontol. 2010 Mar;37(3):230-40.

12. Amann RI, Ludwig W, Schleifer KH. Phylogenetic identification and in situ detection of individual microbial cells without cultivation. Microbiol Rev. 1995 Mar;59(1):143-69.

13. Moter A, Gobel UB. Fluorescence in situ hybridization (FISH) for direct visualization of microorganisms. J Microbiol Methods. $2000 \mathrm{Jul} ; 41(2): 85-112$.
14. Ainamo J, Bay I. Problems and proposals for recording gingivitis and plaque. Int Dent J. 1975 Dec;25(4):229-35.

15. López NJ, Smith PC, Gutierrez J. Higher risk of preterm birth and low birth weight in women with periodontal disease. J Dent Res. 2002 Jan;81(1):58-63.

16. Sunde PT, Olsen I, Gobel UB, Theegarten D, Winter S, Debelian GJ, et al. Fluorescence in situ hybridization (FISH) for direct visualization of bacteria in periapical lesions of asymptomatic root-filled teeth. Microbiology. 2003 May;149(Pt 5):1095-102.

17. Riep B, Edesi-Neuss L, Claessen F, Skarabis H, Ehmke B, Flemmig TF, et al. Are putative periodontal pathogens reliable diagnostic markers?. J Clin Microbiol. 2009 Jun;47(6):170511.

18. Moter A, Hoenig C, Choi BK, Riep B, Göbel UB. Molecular epidemiology of oral treponemes associated with periodontal disease. J Clin Microbiol. 1998 May;36(5):1399-403.

19. Gmür R, Wyss C, Xue Y, Thurnheer T, Guggenheim B. Gingival crevice microbiota from Chinese patients with gingivitis or necrotizing ulcerative gingivitis. Eur J Oral Sci. 2004 Feb;112(1):33-41.

20. Gmür R, Thurnheer T. Direct quantitative differentiation between Prevotella intermedia and Prevotella nigrescens in clinical specimens. Microbiology. 2002 May;148(Pt 5):137987.

21. Cottrell MT, Kirchman DL. Community composition of marine bacterioplankton determined by $16 \mathrm{~S}$ rRNA gene clone libraries and fluorescence in situ hybridization. Appl Environ Microbiol. 2000 Dec;66(12):5116-22.

22. Epstein SS, Rossel J. Enumeration of sandy sediment bacteria: search for optimal protocol. Mar Ecol Prog Ser. 1995;117:28998.

23. Taani DQ, Habashneh R, Hammad MM, Batieha A. The periodontal status of pregnant women and its relationship with socio-demographic and clinical variables. J Oral Rehabil. 2003 Apr;30(4):440-5.

24. Markou E, Eleana B, Lazaros T, Antonios K. The influence of sex steroid hormones on gingiva of women. Open Dent J. 2009 Jun 5;3:114-9.

25. Mealey BL, Moritz AJ. Hormonal influences: effects of diabetes mellitus and endogenous female sex steroid hormones on the periodontium. Periodontol 2000. 2003 Jun;32(1):59-81.

26. Han YW. Oral health and adverse pregnancy outcomes what's next? J Dent Res. 2011 Mar;90(3):289-93. 